\title{
Subjective Satisfaction in Long-term Orthokeratology Patients
}

\author{
Elena Santolaria, M.Sc., Alejandro Cerviño, Ph.D., Antonio Queiros, Ph.D., R. Brautaset, \\ and J. M. González-Méijome, Ph.D.
}

Purpose: To evaluate the subjective satisfaction in visual stability, night vision complains, and light distortion phenomena, and also the number of hours and days per week of lens wear, in patients with myopia undergoing orthokeratology (OK) treatment for at least 1 year.

Methods: A visual analog scale (VAS) questionnaire containing 18 items was administered to 44 patients, 29 women and 15 men (mean age, $24.39 \pm$ 9.11 years), with a baseline spherical equivalent refractive error of $-2.40 \pm$ 0.94 diopters and astigmatism up to -0.5 diopters. Average treatment period by the time of data collection was $19 \pm 7$ months. Patients rated their satisfaction with the correction, with complaints of visual distortion being graded on a scale of 0 to 10 , where 0 indicated no discomfort at all. Results: More than $50 \%$ of patients skipped lens wear at least 1 night per week. The most common wearing pattern was $6-8$ hours a day for $72.7 \%$ with $54.5 \%$ of patients wearing lenses every $2-3$ nights only. Subjective vision scores after lens removal was $9.1 \pm 1.1$ after having worn the lenses and $8.1 \pm 1.4$ after skipping lens wear for 1 night. Subjective vision scores before lens insertion at the end of the day was $6.9 \pm 2.0$ and $5.8 \pm 2.4$, respectively. The number of hours until noticeable blur reduced with increased level of baseline myopia $(\mathrm{r}=0.396 ; P<0.001)$.

Conclusion: Orthokeratology patients show an irregular wearing pattern after 1 year of treatment that has significant effects on the subjective visual performance over the next day of skipping lens wear. Light distortion under low-light conditions seems to be a transient complication of the treatment and most of the patients report an improvement after the first weeks of treatment.

Key Words: corneal refractive therapy-overnight orthokeratologyvisual acuity—-light distortion — subjective satisfaction.

(Eye \& Contact Lens 2013;0: 1-5)

$\mathrm{O}$ rthokeratology (OK) or overnight orthokeratology is a procedure that aims to change the refractive error of the eye by means of changing corneal shape and has become more popular as a nonsurgical method to decrease dependency on daily use of spectacles or conventional contact lenses. ${ }^{1,2}$

From the Private Practice (E.S.), Onda, Castellon, Spain; Department of Optics (A.C.), Optometry Research Group, University of Valencia, Valencia, Spain; Center of Physics (Optometry) (A.Q, J.M.G-M), Universidade do Minho, Braga, Portugal; and Optometry Unit, Department of Clinical Neuroscience (R.B.), Karolinska Institutet, Stockholm, Sweden.

Supported in part by projects PTDC/SAU-BEB/098391/2008//FCOMP01-0124-FEDER-010897 and PTDC/SAU-BEB/098392/2008//FCOMP01-0124-FEDER-010898 funded by Fundação para a Ciência e Tecnologia (FCT) of Portugal.

The authors have no conflicts of interest to disclose.

Address correspondence and reprint requests to Elena Santolaria, M.Sc., Private Practice, C/Falcons, 1 Bajo, 12200 Onda, Castellón, Spain; e-mail: esantolaria@yahoo.es

Accepted XX XX, XXXX.

DOI: 10.1097/ICL.0b013e3182a27777
Clinical results show OK as an effective treatment for the correction of low-to-moderate myopia up to -6.00 diopters (D) of sphere with less than $2.00 \mathrm{D}$ of cylinder. Although the treatment is reversible and regression during the day without lens wear is expected, ${ }^{3}$ some patients are able to keep a reasonable level of visual performance without using their lenses every night. ${ }^{4}$ However, to date, there is no information available from a clinical setting about the prevalence of irregular wearing patterns and potential visual consequences. Moreover, despite the considerable amount of scientific information available about OK outcomes, ${ }^{5}$ most of the studies show short- to medium-term results with few publications reporting long-term results after more than 1 year of treatment.

Thus, the aim of this study was to determine subjective perception of patients wearing OK lenses for more than 1 year with regards to visual outcomes and satisfaction at the beginning and end of the day, and to correlate it with the baseline refractive error.

\section{METHODS}

A total of 44 patients undergoing overnight $\mathrm{OK}$ for myopia correction with reverse geometry rigid gas-permeable contact lenses were recruited at an optometric clinic. Inclusion criteria required that they were successful wearers for at least 1 year. Patients were informed of the nature of the study and signed a consent form after all concerns have been successfully addressed. Following the tenets of the Declaration of Helsinki, the protocol of the study has been reviewed and approved by the University of Valencia. Patients underwent a comprehensive optometric examination and were free of any pathological condition (ocular or systemic), showed satisfactory results on objective and subjective refractive evaluation, and had uncorrected monocular visual acuity (VA) of 0.67 (20/30) or better. Uncorrected VA has been measured under photopic conditions at $5 \mathrm{~m}$ using a decimal scale chart and also reported as Snellen acuity values. Subjective baseline refraction and refraction at the time of data collection were determined as the sphero-cylindrical combination that rendered the best distance VA with the highest positive power. However, VA values are not considered for statistical analysis. According to the protocol followed for overnight orthokeratology patients, all visits were performed in the afternoon between 17:00 and 20:00 after an average of 10 to 12 hours since lens removal. Demographic, refractive, and keratometric data of patients at baseline are presented in Table 1.

\section{Outcome Measures}

Subjective response was recorded in the form of a survey of 18 questions including information about the number of nights wearing the lenses in a week, number of hours of lens wear per 
TABLE 1. Baseline Demographic, Refractive, Keratometric Data (mean $\pm S D$ ), and Range (Minimum and Maximum)

\begin{tabular}{lc}
\hline Age & $24.4 \pm 9.1$ years $(10-46)$ \\
Gender (male/female) & 44 patients $(15 / 29)$ \\
M (baseline) & $-2.40 \pm 0.94 \mathrm{D}(-1.00$ to -4.75$)$ \\
J0 (baseline) & $0.01 \pm 0.26 \mathrm{D}(-0.50$ to 0.49$)$ \\
J45 (baseline) & $-0.01 \pm 0.17 \mathrm{D}(-0.48$ to 0.36$)$ \\
VA (monocular best corrected) & $1.21 \pm 0.23(0.67$ to 2.0$)$ \\
"K"(baseline) & $7.76 \pm 0.29 \mathrm{~mm}(7.20$ to 8.64$)$ \\
Eccentricity (baseline) & $0.46 \pm 0.13(0.06$ to 0.83$)$ \\
\hline
\end{tabular}

VA, visual acuity; D, diopters.

night, number of hours until noticeable blur, and subjective ratings for visual performance under photopic and mesopic conditions using visual analog scale (VAS) including the sensation of visual distortion around sources of light under outdoor conditions (traffic lights, car lamps, street lights, etc) or at home conditions (small sources of light from electronic equipment). Light distortion here refers to effects such as the combination of haloes, glare, and starburst around bright lights under low-lighting conditions. Images found in the Internet simulating these conditions were presented to the wearers to allow them to better understand the light distortion concept. The list of questions is presented as Appendix 1 .

\section{Statistical Analysis}

Statistical analysis was conducted using SPSS software v19.0 (SPSS Inc, Chicago, IL). Normality of data was assessed with Kolmogorov-Smirnov test. Descriptive statistics of the variables measured in the study were produced. Paired-sample $t$ tests were conducted to compare the outcomes after sleeping regularly wearing their lenses with the outcomes after skipping lens wear. Correlation analysis was applied to evaluate the impact of age or baseline spherical equivalent refraction on subjective visual outcomes. When normality could not be assumed, Spearman rho correlation coefficient was calculated.

\section{RESULTS}

All the 44 patients enrolled in the study answered the questionnaire and underwent the optometric examination to confirm that they met the inclusion criteria. Average treatment period at the time of data collection was $19 \pm 7$ months. Uncorrected VA after treatment was $1.21 \pm 0.23$ decimal units (20/16) under photopic monocular conditions and $1.44 \pm 0.22$ decimal units $(20 / 14)$ under photopic binocular conditions. Spherical equivalent residual refraction after a regular wearing pattern (not having skipped lens wear at least in the previous 2 nights) was $-0.16 \pm 0.28 \mathrm{D}[-0.13$ to -0.75$]$ with refractive astigmatism $\leq 0.50 \mathrm{D}$ in all cases. Figure 1 presents the graphical distribution of lens wearing habits.

More than half of the sample population (55\%) reported not wearing the lenses every night of the week as shown in Figure 1. Average wearing frequency was $6.14 \pm 1.17$ days per week, and $55 \%$ of them reported acceptable vision (score higher than 6 ) on the following day after not sleeping on their lenses, whereas, the vision in the evening was worse (score less than 6). A significant trend was found for patients wearing the lenses more nights per week as the baseline level of myopia increased (Spearman rho $=-0.388 ; P=0.041$ ) as shown in Figure 2 .

The results of the survey are summarized in Table 2 for questions evaluated using VAS and other questions recorded as continuous

\section{Nights per week of lens wear}

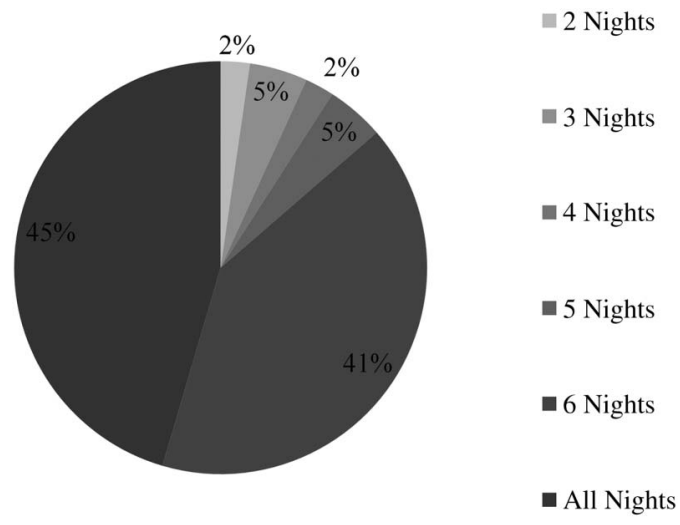

FIG. 1. Number of nights per week that the patients use to wear lenses overnight.

variables and in Table 3 for categorical variables. The reported overnight wearing time was $6-8$ hours for $72.7 \%$ and $8-10$ hours for $22.73 \%$ of the participants. Table 2 also shows the correlation between the scores of the continuous variables in the questionnaire and the patient's binocular VA, age, and baseline spherical equivalent refraction and VA at the time of data collection. After skipping lens wear, subjective vision score was significantly decreased compared with the reported vision scores when lenses are worn regularly. Differences were statistically significant (paired $t$ test, $P<0.05$ ) in the morning after lens removal (from 9.1 to 6.9) and in the evening before lens insertion (from 8.1 to 5.8 ). Visual acuity was significantly correlated with vision of fine details at the end of the day after having skipped lens wear $(\mathrm{r}=0.335 ; P=0.026)$. The overall score seeing fine details at the end of the day, after skipping 1 night of lens wear, was $5.8 \pm 2.4$ points (range, 0 to 10 ). Visual acuity was significantly correlated with vision at the end of the following day after skipping lens wear for 1 night $(\mathrm{r}=0.372 ; P=0.020)$. Visual acuity was significantly correlated with time (hours) until noticeable worsening of vision after skipping lens wear $(r=0.425 ; P=0.007)$.

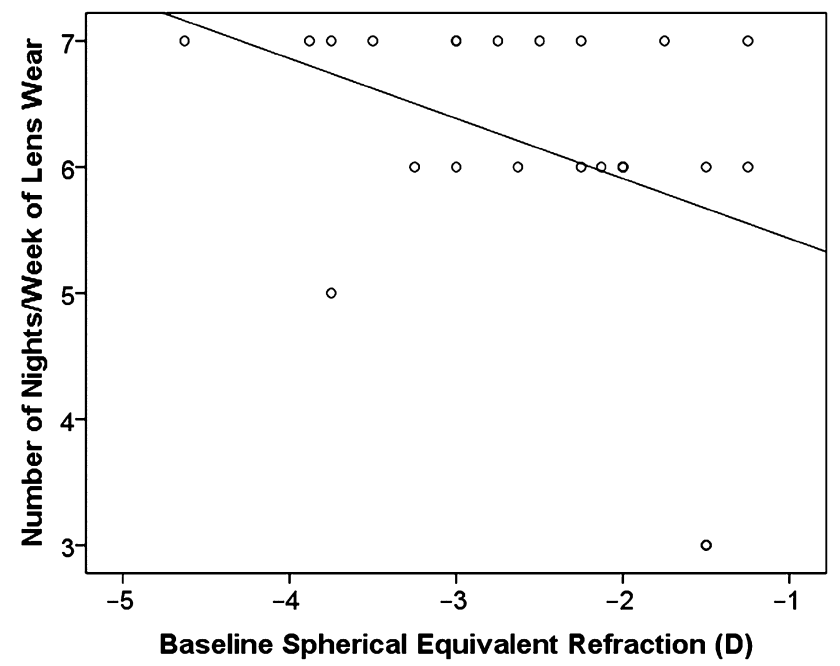

FIG. 2. Scatterplot displaying the correlation between spherical equivalent refraction at baseline and the nights per week of lens wear (rho $=-0.388 ; P=0.041 ; \mathrm{n}=44$ ). $\mathrm{D}$, diopters. 
TABLE 2. Descriptive Statistics (Mean \pm SD and Range) for Outcomes From the Survey Graded Using Visual Analog Scale for Continuous Variables Along With Correlations With Spherical Equivalent Refraction, Age, and Visual Acuity

\begin{tabular}{|c|c|c|c|c|c|c|c|}
\hline \multirow[b]{3}{*}{ Question } & \multirow[b]{3}{*}{ Mean \pm SD (Min, Max) } & \multicolumn{6}{|c|}{ Subjective Response vs. } \\
\hline & & \multicolumn{2}{|c|}{ Baseline Rx } & \multicolumn{2}{|c|}{ Age } & \multicolumn{2}{|l|}{ VA } \\
\hline & & $r$ (Pearson) & $P$ & r (Pearson) & $P$ & r (Pearson) & $P$ \\
\hline 5-Comfort at lens insertion & $7.8 \pm 1.4(3,10)$ & -0.262 & 0.086 & 0.102 & 0.512 & -0.127 & 0.411 \\
\hline 6-Comfort at lens removal & $7.1 \pm 2.3(1,10)$ & -0.168 & 0.277 & -0.007 & 0.965 & -0.104 & 0.501 \\
\hline 7-Vision of fine details at the end of the day & $8.1 \pm 1.4(4,10)$ & 0.270 & 0.076 & 0.030 & 0.848 & 0.229 & 0.134 \\
\hline 8-Vision of fine details after lens removal having slept on them & $9.1 \pm 1.1(6,10)$ & -0.084 & 0.586 & -0.158 & 0.305 & 0.335 & $0.026^{*}$ \\
\hline 9-Luminous distortion around car lights while driving at night & $7.3 \pm 2.8(1,10)$ & -0.136 & 10.377 & -0.113 & 0.467 & 0.132 & 0.393 \\
\hline 12-Luminous distortion at home & $8.2 \pm 2.3(2,10)$ & -0.070 & 0.650 & -0.268 & 0.079 & 0.165 & 0.283 \\
\hline 15-Vision on awakening after skipping wear & $6.9 \pm 2.0(2,10)$ & 0.277 & 0.088 & 0.044 & 0.792 & 0.155 & 0.346 \\
\hline 16-Vision at end of day after skipping wear & $5.8 \pm 2.4(1,10)$ & 0.543 & $<0.001^{*}$ & -0.069 & 0.678 & 0.372 & $0.020 *$ \\
\hline
\end{tabular}

*Statistically significant.

Min, minimum; Max, maximum.

There was an inverse correlation between the baseline spherical equivalent refraction and the number of hours until noticeable blur F3 after having skipped lens wear the previous night (Figure 3A). Subjective visual sensation at the end of the day was poorer for patients with higher myopia if they did not wear their lenses on the previous night (Figure 3B).

Regarding the distortion observed around bright lights at home under low-light conditions (Questions 12 to 14), 45.5\% did not report complains at all; whereas, $70.8 \%$ of those reporting complains scored this sensation as 1 or 2 points, and $16.7 \%$ scored it within the range of 6 to 9 points (the higher the score, the more significant the complain was). About 36.\% reported that they still had a certain degree of light distortion while driving at night after 1 year of lens wear; whereas, $18.2 \%$ reported a reduction in sensation of light distortion throughout the year of lens wear.

Light distortion sensation around car lights at night (Questions 9 to 11 ) was not present for $45.5 \%$ of the subjects after 1 year, decreased for $22.7 \%$ of patients, and $25.0 \%$ reported that it remained unchanged, and $2.3 \%$ reported an increase since they started wearing lens. Patients reported that it took an average of $14.63 \pm 35.29$ weeks to notice a decrease in the sensation of luminous distortion around car lights and $10.1 \pm 14.4$ weeks average for light distortion experience at home. There was a positive correlation between the age of patients and the time to notice a reduction of light distortion around car lights under dim illumination $(\mathrm{r}=0.749 ; P=0.005)$ with older patients presenting a trend

\section{DISCUSSION}

Orthokeratology is relatively new alternative technique for the compensation myopia, compared with other more conventional

methods of correction such as glasses or soft contact lenses, which allows users to correct the refractive error during sleep, getting a good quality of vision during the day without the aid of any other method of visual correction. ${ }^{2}$ Despite the increasing interest in overnight $\mathrm{OK}$, clinical information regarding the actual pattern of lens wear by these patients in the long-term and their subjective satisfaction with their vision has been scarce.

It is well accepted that during this treatment, there are changes in AU9 corneal aberrations that have an impact in the optical quality of the eye, and thus the visual function, ${ }^{6-8}$ this is because of the modification of the original shape of the anterior corneal surface. Light distortion in the form of haloes usually reported by OK patients in clinical practice might be linked with such changes. Previous studies concluded that halos are significantly correlated with corneal irregularity, and this parameter alone would justify the performance of night vision in patients treated with OK. ${ }^{9}$ Although we are not reporting topographic data, it is well known that orthokeratology is associated with significant changes in corneal topography. Queirós et al. ${ }^{10}$ compared the front surface corneal aberrations of 3 different techniques to correct low-to-moderate myopia, namely laser in situ keratomileusis (LASIK), custom LASIK, and OK. There was a significant increase in corneal aberrations, which was more exacerbated for OK for pupils larger than $5 \mathrm{~mm}$. Orthokeratology induced higher values of spherical aberration compared to standard and custom LASIK. The correlation between increase in spherical aberration and haloes has been previously pointed by Villa et al. ${ }^{11}$ in a study involving successful LASIK treatments. The authors found a moderate correlation between spherical corneal aberrations and halo disturbance index. However, the absence of aberration measurement and the lack of pupil size measurements does not allow us to evaluate the potential involvement of front surface corneal aberrations on light distortion sensation.

TABLE 3. Frequencies (\%) for Categorical Questions Included in the Survey Regarding Time of Wear or Time Until Noticing Vision Changes

\begin{tabular}{|c|c|c|c|c|c|}
\hline Question & 2 to $6 \mathrm{hrs}, \%$ & 4 to 6 hrs, $\%$ & 6 to $8 \mathrm{hrs}, \%$ & 8 to $10 \mathrm{hrs}, \%$ & Not noticeable, \% \\
\hline 1-Hours until noticeable blurred vision & & & 4.55 & 9.09 & 86.36 \\
\hline 2-Hours until insufficient vision & & & 2.30 & 2.30 & 95.40 \\
\hline 4-Hours of overnight use & & 4.60 & 72.70 & 22.70 & \\
\hline 17-Hours until blurred vision after skipping lens wear & 15.40 & 20.50 & 20.50 & 23.10 & 20.50 \\
\hline 18-Hours until insufficient vision after skipping lens wear & 5.13 & 15.38 & 10.26 & 23.08 & 46.15 \\
\hline
\end{tabular}




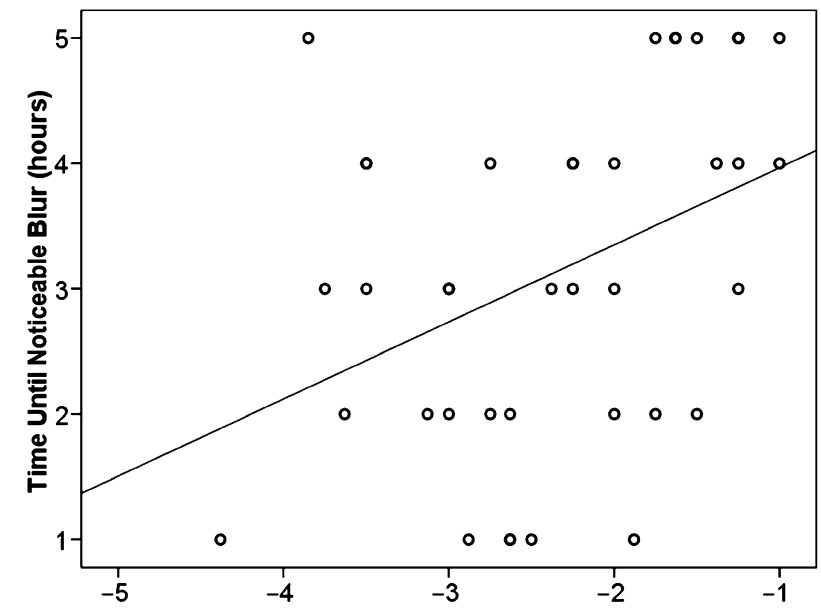

A Baseline Spherical Equivalent Refraction (D)

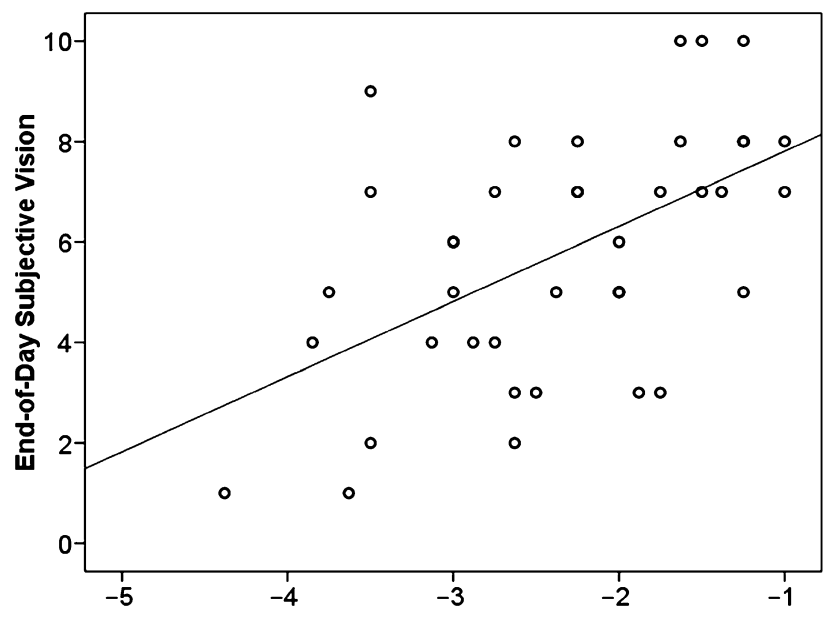

B

Baseline Spherical Equivalent Refraction (D)

FIG. 3. Correlation between the baseline spherical equivalent refractive error (diopters) and the number of hours until noticeable blur $(A), r=0.396 ; P=0.013$, and subjective visual performance at the end of the day after skipping lens wear for 1 night $(B), r=0.543$; $P<0.001$. D, diopters.

Despite the limitations of $\mathrm{OK}$ as a reversible procedure to correct low-to-moderate refractive error, previous studies have shown that most patients will prefer OK to soft contact lens. ${ }^{12}$ More recently, Queirós et al. ${ }^{13}$ have demonstrated that OK compares to other methods of visual correction and outperformed spectacle and soft contact lens correction when a global score of the NEI-RQL42 questionnaire was analyzed. Aspects such as glare experience were rated lowest for $\mathrm{OK}$, which might be related to the changes in corneal shape. Furthermore, even though the procedure is reversible and needs regular overnight contact lens wear, it is known from the clinical setting that not every patient wears the lenses on a regular basis, and thus the symptoms might exacerbate. These known facts draw the attention to the subjective perception of the individual about the procedure.

The results of this study show that a significant number of patients do not wear their contact lenses on a regular basis throughout the week. The observed lower scores in subjective vision after skipping lens wear seem to be overcome by the

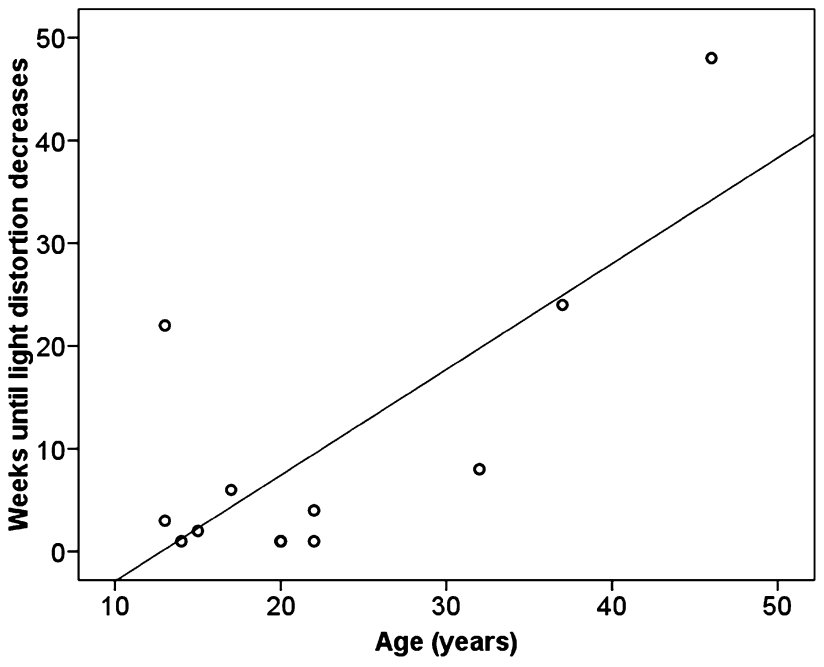

FIG. 4. Scatterplot displaying the correlation between age and the number of weeks it takes for light distortion perception to decrease $(r=0.749 ; P=0.005)$.

convenience of not having to wear the lenses on a regular basis. Assuming that changes in the ocular surface are maintained in the medium and long-term, ${ }^{8}$ it is interesting to observe that the light distortion symptoms in the form of haloes and other sensations disappear for most of the patients within several weeks from the initiation of treatment. This might reflect some form of neuroadaptation to the perception of such distortions. As expected, the time to adapt tends to be longer for higher refractive errors and might be somewhat related to the age of the patient such that it takes longer for older patients to adapt. However, data presented in Figure 4 do not allow to take this as a clinical rule. The age range of our patients is narrow, and there is a considerable scatter of data. The present cohort excludes patients with ocular opacities that might increase light scattering and produce some forms of light distortion symptoms in the form of haloes and other sensations. Therefore, this trend might be the result of the older patients having slightly higher myopia than younger patients in the sample, as a slower response to orthokeratology by older patients has been noted previously. ${ }^{14}$

The information presented in this study is relevant for the clinician to manage the information given to the patient and avoid creating unrealistic expectations in visual quality for the first periods of treatment. The clinician may recognize that the patient is likely to experience some forms of light distortion, both at home and while driving at night, during the initial phases of the treatment. The time to adapt to such visual symptoms will differ between individuals, but the present results suggest that lower myopes will adapt faster. A limitation of the study is the absence of pupil size measurements, which might help to explain the differences in light distortion between individuals. Despite this limitation, data of pupil size recorded with the corneal topographer at baseline was analyzed and did not show a correlation with the intensity of halo and light distortion sensation.

The results of this study show that most of the complains decrease with time. After 1 year of follow up, only a small percentage of $\mathrm{OK}$ patients report persistent significant complains related with light distortion under low light conditions. 
In summary, the present results show that patient satisfaction with visual outcomes is high and improves over the first year of treatment. Symptoms of light distortion are present as expected, considering the morphological changes induced by the treatment to the anterior corneal surface, although this study does not allow to establish a causative relationship between subjective complains and optical quality changes. Although the prescription guidelines require lens wear every night to retain the correction effect, a significant number of patients skip several nights of lens wear a week while still retaining a satisfactory subjective visual quality. Time until noticeable blur after skipping lens wear decreased as baseline refractive error increased.

\section{REFERENCES}

1. Soni PS, Nguyen TT, Bonanno JA. Overnight orthokeratology: Visual and corneal changes. Eye Contact Lens 2003;29:137-145.

2. Nichols JJ, Marsich MM, Nguyen M, et al. Overnight orthokeratology. Optom Vis Sci 2000;77:252-259.

3. Soni PS, Nguyen TT, Bonanno JA. Overnight orthokeratology: Refractive and corneal recovery after discontinuation of reverse-geometry lenses. Eye Contact Lens 2004;30:254-262.

4. Swarbrick HA, Wong G, O'Leary DJ. Corneal response to orthokeratology. Optom Vis Sci 1998;75:791-799.

5. Tahhan N, Du Toit R, Papas E, et al. Comparison of reverse-geometry lens designs for overnight orthokeratology. Optom Vis Sci 2003;80: 796-804.
6. Hiraoka $\mathrm{T}$, Okamoto $\mathrm{C}$, Ishii $\mathrm{Y}$, et al. Contrast sensitivity function and ocular higher-order aberrations following overnight orthokeratology. Invest Ophthalmol Vis Sci 2007:48:550-556.

7. Hiraoka T, Okamoto C, Ishii Y, et al. Mesopic contrast sensitivity and ocular higher-order aberrations after overnight orthokeratology. Am J Ophthalmol 2008;145:645-655.

8. Hiraoka T, Mihashi T, Okamoto C, et al. Influence of induced decentered orthokeratology lens on ocular higher-order wavefront aberrations and contrast sensitivity function. J Cataract Refract Surg 2009;35:1918-1926.

9. Kojima T, Hasegawa A, Hara S, et al. Quantitative evaluation of night vision and correlation of refractive and topographical parameters with glare after orthokeratology. Graefes Arch Clin Exp Ophthalmol 2011;249:1519-1526.

10. Queirós A, Villa-Collar C, González-Méijome JM, et al. Effect of pupil size on corneal aberrations before and after standard laser in situ keratomileusis, custom laser in situ keratomileusis, and corneal refractive therapy. $\mathrm{Am} \mathrm{J}$ Ophthalmol 2010;150:97-109.e1.

11. Villa C, Gutierrez R, Jimenez JR, et al. Night vision disturbances after successful LASIK surgery. Br J Ophthalmol 2007;91: 1031-1037.

12. Lipson MJ, Sugar A, Musch DC. Overnight corneal reshaping versus soft daily wear: A visual quality of life study (interim results). Eye Contact Lens 2004;30:214-217.

13. Queirós A, Villa-Collar C, Gutiérrez AR, et al. Quality of life of myopic subjects with different methods of visual correction using the NEI RQL-42 questionnaire. Eye Contact Lens 2012;38:116-121.

14. Jayakumar J, Swarbrick HA. The effect of age on short-term orthokeratology. Optom Vis Sci 2005;82:505-511.

\section{APPENDIX 1: Questionnaire Filled by the} Patients 